\title{
Modified Geometric Distribution of Certain Order and Some of its Properties
}

\author{
Okoli, O.C. \\ Department of Mathematics, Chukwuemeka Odumegwu Ojukwu University, Uli Campus Anambra State Nigeria \\ *Corresponding author's email: odicomatics@yahoo.com,odico2003@yahoo.com
}

\begin{abstract}
In this research work, we present a new distribution function that modifies and generalize the geometric distribution of order $k$ which was introduced by Philippou et al. (1983). The associated statistics, generating function, and its relationship with some other discrete distributions are established. The results obtained in this paper improve, generalize and complement the works of several authors in the literature.
\end{abstract}

\section{Introduction and Preliminaries}

$\mathrm{P}$ hilippou et al. (1983) introduced the distribution of the number of trials until the first occurrence of consecutive $k$ successes in Bernoulli trials with success probability $p$ which they called a negative binomial distribution of order $k$. This distribution have caught the interested of researchers, owning to the work of Philippou et al., (1983) whose contribution in this field seems to be very important. Philippou et al. (1984) studied a negative binomial distribution of order $k$ and defined a Poisson distribution of order $k$, attention has been paid to interrelationships among the so called discrete distributions of order $k$ and their exact distribution theory has been developed extensively by many researchers. For any fixed positive integer $k$, let $N_{k}$ denote the number of independent trials with success probability $p(q=1-p)$ until the first occurrence of consecutive $k$ successes. Philippou gave the following definition for the probability mass function of the event $\left\{N_{k}=x\right\}$ (where $\left\{N_{1}=x\right\}$ reduces to the standard geometric distribution);

Definition 1.1 random variable $N_{k}$ is said to have a geometric distribution of order $k$ with parameter $p$ if the probability mass function $(p m f)$ is given by

$$
\begin{gathered}
f_{k}(x)=P\left(N_{k}=x\right)=\sum_{\begin{array}{c}
x_{1}, x_{2}, \ldots, x_{k} \\
x_{1}+2 x_{2}+\cdots+k x_{k}=x-k
\end{array}}\left(\begin{array}{c}
x_{1}+x_{2}+\cdots+x_{k} \\
x_{1}, x_{2}, \ldots, x_{k}
\end{array}\right) \times \\
p^{x}\left(\frac{q}{p}\right)^{x_{1}+x_{2}+\cdots+x_{k}} ; x \geq k
\end{gathered}
$$

Where the summation is taken over all non-negative integers $x_{1}, x_{2}, \ldots, x_{k}$ satisfying the condition $x_{1}+2 x_{2}+\cdots+k x_{k}=$ $x-k$.

Ever since then, related concepts has been introduced, reliability theory of the consecutive- $k$-out-of- $n$ :F systems and studied by Kontoleon (1980), Chiang and Niu (1981) and Derman, Lieberman and Ross (1982). Several related concepts on discrete distributions of order $k$ were studied by Lambiris and Papastavridis (1985), Fu (1985;1986a;1986b), Fu and Beihua (1987), Aki 
(1985), Hirano (1986), Philippou (1986), Papastavridis (1987), Aki and Hirano (1988; 1989; 1996; 1997), Chrysaphinou and Papastavridis (1990c), Fu and Koutras (1994a;1994b), Godbole (1990a; 1991), Griffith (1983), Hirano and Aki (1993), Charalambides (1994) and Makri and Philippou (1994), Feller (1968). Various modifications have been made also on the underlying sequence, e.g. replacing the Bernoulli trials for other random sequences such as some urn models, a binary sequence of order $k$ as in Aki (1985), Aki and Hirano (1994;1995), Dhar and Jiang (1995) and Balakrishnan (1997). Recently, Shao and $\mathrm{Fu}$ (2015) derived the same distribution using the transition probability flow graphs (TPFG) method.

It is quite interesting how the classical geometric distribution and related discrete distributions has been generalized and developed by above authors. However, these generalizations of classical geometric distribution is by no mean exhaustive, hence, in this research work, we introduce a new distribution function for a class of arbitrary sequence of $S, F$ (where $\mathrm{S}$ and $\mathrm{F}$ means success and failure) events that modifies and generalize the geometric distribution of order $k$. Now, let $E_{x}\left(i_{m}, j_{m}\right)$ be a sequence of $S, F$ (binary) events that terminate at the $x^{t h}$ trial in which repetition is allowed. If we suppose that this terminal point, is the point at which the event of interest occurs (success). For a given $x^{\text {th }}$ terminal point of trials in which the event of interest occurs, observe that this sequence of event forms a partition on $x$. Suppose $E_{x}\left(i_{m}, j_{m}\right)$ partitioned $x$ into $k$-parts such that $E_{x}\left(i_{m}, j_{m}\right)$ repeat itself $x_{m}$ time $(m=1,2, \ldots, k+1)$ then we consider the sequence of events $\left\{E_{x}\left(i_{m}, j_{m}\right)\right\}_{x=1}^{\infty}$ with support on $M$ define as follows

$$
E_{x}\left(i_{m}, j_{m}\right)=\left(S^{i_{m}} F^{j_{m}}\right)^{x_{m}} ; i_{m}, j_{m} \geq 0 ; m \in M, \quad x \geq 1
$$

Where $i_{m}, j_{m}$ are non-negative integer functions of $m$, with support on $M=\{1,2, \ldots, k+1\}$.

Definition 1.2 Let $N_{i_{k+1}, j_{k+1}}$ be a random variable that counts the number of trials until the occurrence of event of interest $E_{x}\left(i_{k+1}, j_{k+1}\right)$ in $(1.2)$ with success probability $p(q=1-p)$, then we say that $N_{i_{k+1}, j_{k+1}}$ follows a modified geometric distribution of order $\left(i_{k+1}, j_{k+1}\right)$ denoted by $M G_{i_{k+1}, j_{k+1}}(. ; p)$ if the probability mass function $(p m f)$ is given by

$$
\begin{aligned}
& \sum_{x_{1}, x_{2}, \ldots, x_{k} ; i_{1}, i_{2}, \ldots, i_{k} ; j_{1}, j_{2}, \ldots, j_{k} \ni}^{f_{i_{k+1}, j_{k+1}}(x)=P\left(\begin{array}{c}
\left.N_{i_{k+1}, j_{k+1}}=x\right)= \\
x_{1}+x_{2}+\cdots+x_{k} \\
x_{1}, x_{2}, \ldots, x_{k}
\end{array}\right) \times} \\
& \sum_{m=1}^{k} i_{m} x_{m}+\sum_{m=1}^{k} j_{m} x_{m}=x-i_{k+1}-j_{k+1} \\
& p^{x}\left(\frac{q}{p}\right)^{\sum_{m=1}^{k} j_{m} x_{m}+j_{k+1}} ; x \geq i_{k+1}+j_{k+1}
\end{aligned}
$$

Where the summation is taken over all non-negative integers $x_{1}, x_{2}, \ldots, x_{k} ; i_{1}, i_{2}, \ldots, i_{k} ; j_{1}, j_{2}, \ldots, j_{k}$ satisfying the condition

$\sum_{m=1}^{k} i_{m} x_{m}+\sum_{m=1}^{k} j_{m} x_{m}=x-i_{k+1}-j_{k+1} ; p^{i_{k+1}} q^{j_{k+1}}=1-\sum_{m=1}^{k} p^{i_{m}} q^{j_{m}}$

Then we say that $f_{i_{k+1}, j_{k+1}}$ is a geometric distribution of order $\left(i_{k+1}, j_{k+1}\right)$.

Is easy to see that (1.3) is a proper probability distribution, and moreover, is general than (1.1). To see this, if we take a particular case of (1.2) and define

$$
j_{m}=\left\{\begin{array}{l}
1, m=1,2, \ldots, k \\
0, m \geq k+1
\end{array} \text { and } \quad i_{m}=m-1\right.
$$

So that (1.2) becomes

$$
E(m-1,1)=S^{m-1} F ; m=1,2, \ldots, k \text { and } E(k, 0)=S^{k}
$$

If we substitute the definitions for $i_{m}$ and $j_{m}$ in (1.4) into (1.3), we easily obtain (1.1) which are results due to Philippou $e t$ al. (1983).

Theorem 1.3 The probability generating function $(p . g . f$.$) of N_{i_{k+1}, j_{k+1}}$ distributed as $M G_{i_{k+1}, j_{k+1}}(. ; p)$ is given by

Where $\pi_{m}=p^{i_{m}} q^{j_{m}}$ and $\delta_{m}=i_{m}+j_{m}$

$$
G_{N_{i_{k+1}, j_{k+1}}}(t)=\frac{\pi_{k+1} t^{\delta_{k+1}}}{1-\sum_{m=1}^{k} \pi_{m} t^{\delta_{m}}} ;|t| \leq 1
$$

Proof.

$$
\begin{aligned}
& =\sum_{x=i_{k+1}+j_{k+1}}^{\infty} t^{x} P\left(N_{i_{k+1}, j_{k+1}}=x\right) \\
& =\sum_{x=i_{k+1}+j_{k+1}}^{\infty} \sum_{x_{1}, x_{2}, \ldots, x_{k} ; i_{1}, i_{2}, \ldots, i_{k} ; j_{1}, j_{2}, \ldots, j_{k} \ni}\left(\begin{array}{c}
x_{1}+x_{2}+\cdots+x_{k} \\
x_{1}, x_{2}, \ldots, x_{k}
\end{array}\right) \times \\
& \sum_{m=1}^{k} i_{m} x_{m}+\sum_{m=1}^{k} j_{m} x_{m}=x-i_{k+1}-j_{k+1} \\
& p^{x}\left(\frac{q}{p}\right)^{\sum_{m=1}^{k} j_{m} x_{m}+j_{k+1}}
\end{aligned}
$$




$$
\begin{aligned}
& =\sum_{x=0}^{\infty} \sum_{x_{1}, x_{2}, \ldots, x_{k} ; i_{1}, i_{2}, \ldots, i_{k} ; j_{1}, j_{2}, \ldots, j \ni}\left(\begin{array}{c}
x_{1}+x_{2}+\cdots+x_{k} \\
x_{1}, x_{2}, \ldots, x_{k}
\end{array}\right)(p t)^{x+i_{k+1}+j_{k+1}}\left(\frac{q}{p}\right)^{\sum_{m=1}^{k} j_{m} x_{m}+j_{k+1}} \\
& \sum_{m=1}^{k} i_{m} x_{m}+\sum_{m=1}^{k} j_{m} x_{m}=x \\
& =(p t)^{i_{k+1}+j_{k+1}}\left(\frac{q}{p}\right)^{j_{k+1}} \sum_{x=0}^{\infty} \sum_{\begin{array}{c}
x_{1}, x_{2}, \ldots, x_{k} \\
x_{1}+x_{2}+\cdots+x_{k}=x
\end{array}}\left(\begin{array}{c}
x_{1}+x_{2}+\cdots+x_{k} \\
x_{1}, x_{2}, \ldots, x_{k}
\end{array}\right) \times \\
& (p t)^{\sum_{m=1}^{k} i_{m} x_{m}+\sum_{m=1}^{k} j_{m} x_{m}}\left(\frac{q}{p}\right)^{\sum_{m=1}^{k} j_{m} x_{m}} \\
& =(p t)^{i_{k+1}}(q t)^{j_{k+1}} \sum_{x=0}^{\infty} \sum_{\begin{array}{c}
x_{1}, x_{2}, \ldots, x_{k} \\
x_{1}+x_{2}+\cdots+x_{k}=x
\end{array}}\left(\begin{array}{c}
x_{1}+x_{2}+\cdots+x_{k} \\
x_{1}, x_{2}, \ldots, x_{k}
\end{array}\right)(p t)^{\sum_{m=1}^{k} i_{m} x_{m}}(q t)^{\sum_{m=1}^{k} j_{m} x_{m}} \\
& =(p t)^{i_{k+1}}(q t)^{j_{k+1}} \sum_{x=0}^{\infty}\left(\sum_{m=1}^{k}(p t)^{i_{m}}(q t)^{j_{m}}\right)^{x} \\
& =\frac{(p t)^{i_{k+1}}(q t)^{j_{k+1}}}{1-\sum_{m=1}^{k}(p t)^{i_{m}}(q t)^{j_{m}}}=\frac{\pi_{k+1} t^{\delta_{k+1}}}{1-\sum_{m=1}^{k} \pi_{m} t^{\delta_{m}}} \quad ;|t| \leq 1
\end{aligned}
$$

Observe that $M G_{i_{k+1}, j_{k+1}}(. ; p)$ is a proper probability distribution, which can be easily deduced from (1.7).

Corollary 1.3 Let $G_{N_{i_{k+1}}, j_{k+1}}(t)$ be as in theorem 1.3, if in particular we define

Then

$$
j_{m}=\left\{\begin{array}{l}
1, m=1,2, \ldots, k \\
0, m \geq k+1
\end{array} \text { and } \quad i_{m}=m-1\right.
$$

$$
G_{N_{k, 0}}(t)=\frac{(p t)^{k}(1-p t)}{1-t+q t(p t)^{k}}
$$

Equation (1.8) has been obtained by Feller (1968), Philippou et al. (1983), Aki (1994), Shao and Wang (2015) via different approach. In the remaining part of this work, the results of our corollaries are result obtained by some of the above mentioned authors.

Theorem 1.4 Let $N_{i_{k+1}, j_{k+1}}$ be as in definition 1.2. Then

$$
\begin{gathered}
E\left(N_{i_{k+1}, j_{k+1}}\right)=\delta_{k+1}+\frac{\sum_{m=1}^{k} \delta_{m} \pi_{m}}{\pi_{k+1}} \\
\operatorname{Var}\left(N_{i_{k+1}, j_{k+1}}\right)=\frac{\sum_{m=1}^{k} \delta_{m}\left(\delta_{m}-1\right) \pi_{m}}{\pi_{k+1}}+\left(1-3 \delta_{k+1}\right) \frac{\sum_{m=1}^{k} \delta_{m} \pi_{m}}{\pi_{k+1}}-\delta_{k+1}{ }^{2} \\
-\left(\frac{\sum_{m=1}^{k} \delta_{m} \pi_{m}}{\pi_{k+1}}\right)^{2}
\end{gathered}
$$

Where $\pi_{m}=p^{i_{m}} q^{j_{m}}$ and $\delta_{m}=i_{m}+j_{m}$

\section{Proof.}

(i) It is well known that $E\left(N_{i_{k+1}, j_{k+1}}\right)=\left.\frac{\partial G_{N_{i_{k+1}, j_{k+1}}}(t)}{\partial t}\right|_{t=1}$. But

$$
\begin{gathered}
\frac{\partial G_{N_{i_{k+1}} j_{k+1}}(t)}{\partial t} \\
=\left(i_{k+1}+j_{k+1}\right) p^{i_{k+1}} q^{j_{k+1}} t^{i_{k+1}+j_{k+1}-1}\left(1-\sum_{m=1}^{k} p^{i_{m}} q^{j_{m}} t^{i_{m}+j_{m}}\right)^{-1}+ \\
p^{i_{k+1}} q^{j_{k+1}} t^{i_{k+1}+j_{k+1}}\left(1-\sum_{m=1}^{k} p^{i_{m}} q^{j_{m}} t^{i_{m}+j_{m}}\right)^{-2} \times \\
\left(\sum_{m=1}^{k}\left(i_{m}+j_{m}\right) p^{i_{m}} q^{j_{m}} t^{i_{m}+j_{m}-1}\right) \\
=\delta_{k+1} t^{-1}+\frac{\sum_{m=1}^{k} \delta_{m} \pi_{m} t^{\delta_{m}-1}}{\pi_{k+1} t^{\delta_{m}}}
\end{gathered}
$$

Hence, we have that

$$
\left.\frac{\partial G_{N_{i_{k+1}, j_{k+1}}}(t)}{\partial t}\right|_{t=1}=\delta_{k+1}+\frac{\sum_{m=1}^{k} \delta_{m} \pi_{m}}{p^{i_{k+1}} q^{j_{k+1}}}
$$


(ii)

Observe that

Thus,

$$
\begin{gathered}
\frac{\partial^{2} G_{N_{i_{k+1}, j_{k+1}}}(t)}{\partial t^{2}} \\
=-\delta_{k+1} t^{-2}+\frac{\sum_{m=1}^{k} \delta_{m}\left(\delta_{m}-1\right) \pi_{m} t^{\delta_{m}-2}}{\pi_{k+1} t^{\delta_{k+1}}}-\frac{\delta_{k+1} \pi_{k+1} t^{\delta_{k+1}-1} \sum_{m=1}^{k} \delta_{m} \pi_{m} t^{\delta_{m}-2}}{\left(\pi_{k+1} t^{\delta_{k+1}}\right)^{2}}
\end{gathered}
$$

$$
\begin{gathered}
\left.\frac{\partial^{2} G_{N_{i_{k+1}, j_{k+1}}}(t)}{\partial t^{2}}\right|_{t=1} \\
=-\delta_{k+1}+\frac{\sum_{m=1}^{k} \delta_{m}\left(\delta_{m}-1\right) \pi_{m}}{\pi_{k+1}}-\frac{\delta_{k+1} \sum_{m=1}^{k} \delta_{m} \pi_{m}}{\pi_{k+1}}
\end{gathered}
$$

Using the result in (i) above and after some algebraic simplification, it follows that

$$
\begin{array}{r}
\operatorname{Var}\left(N_{i_{k+1}, j_{k+1}}\right)=\left.\frac{\partial^{2} G_{N_{i_{k+1}} j_{k+1}}(t)}{\partial t^{2}}\right|_{t=1}+\left.\frac{\partial G_{N_{i_{k+1}} j_{k+1}}(t)}{\partial t}\right|_{t=1}-\left(\left.\frac{\partial G_{N_{i_{k+1}} j_{k+1}}(t)}{\partial t}\right|_{t=1}\right)^{2} \\
=\frac{\sum_{m=1}^{k} \delta_{m}\left(\delta_{m}-1\right) \pi_{m}}{\pi_{k+1}}+\left(1-3 \delta_{k+1}\right) \frac{\sum_{m=1}^{k} \delta_{m} \pi_{m}}{\pi_{k+1}}-\delta_{k+1}{ }^{2}-\left(\frac{\sum_{m=1}^{k} \delta_{m} \pi_{m}}{\pi_{k+1}}\right)^{2}
\end{array}
$$

Observe that by theorem 1.4, $E\left(N_{k, 0}\right)=k+\frac{q}{p^{k}}\left[\frac{\left[1-p^{k}-k p^{k}+k p^{k+1}\right]}{(1-p)^{2}}\right]$ is a special case, hence we state the following corollary.

Corollary 1.5 Let $N_{i_{k+1}, j_{k+1}}$ be as in definition 1.2. If in particular we define

Then

$$
j_{m}=\left\{\begin{array}{l}
1, m=1,2, \ldots, k \\
0, m \geq k+1
\end{array} \text { and } \quad i_{m}=m-1\right.
$$

(ii) $\quad \operatorname{Var}\left(N_{k, 0}\right)=\frac{\left[1-(2 k+1) q p^{k}-p^{2 k+1}\right]}{q^{2} p^{k}}$

Definition 1.6 Let $N^{1} i_{k+1}, j_{k+1}, N^{2}{ }_{i_{k+1}, j_{k+1}}, \ldots, N^{r}{ }_{i_{k+1}, j_{k+1}}$ be independent identically distributed random variables. Then the distribution of $N_{i_{k+1}, j_{k+1, r}}=N^{1}{ }_{i_{k+1}, j_{k+1}}+N^{2}{ }_{i_{k+1}, j_{k+1}}+\cdots+N^{r}{ }_{i_{k+1}, j_{k+1}}$ is called a modified negative binomial distribution of order $\left(i_{k+1}, j_{k+1}\right)$ denoted by $M N B_{i_{k+1}, j_{k+1}}(. ; p)$

The following theorem is a consequence of definition 1.6.

Theorem 1.7 Let $N^{1} i_{k+1}, j_{k+1}, N^{2} i_{k+1}, j_{k+1}, \ldots, N^{r}{ }_{i_{k+1}, j_{k+1}}$ be independent identically distributed random variables. Then the probability generating function of $N_{i_{k+1}, j_{k+1}, r}=N^{1}{ }_{i_{k+1}, j_{k+1}}+N^{2}{ }_{i_{k+1}, j_{k+1}}+\cdots+N^{r}{ }_{i_{k+1}, j_{k+1}}$

Where $\pi_{m}=p^{i_{m}} q^{j_{m}}, \delta_{m}=i_{m}+j_{m}$.

$$
G_{N_{i_{k+1}, j_{k+1, r}}}(t)=\left(\frac{\pi_{k+1} t^{\delta_{k+1}}}{1-\sum_{m=1}^{k} \pi_{m} t^{\delta_{m}}}\right)^{r} ;|t| \leq 1
$$

\section{Proof.}

Since $N^{1}{ }_{i_{k+1}} j_{k+1}, N^{2}{ }_{i_{k+1}, j_{k+1}}, \ldots, N^{r}{ }_{i_{k+1}}, j_{k+1}$ are independent identically distributed random variables with p.g.f. $G_{N^{j}} i_{k+1}, j_{k+1}($ as in (1.6)) for each $j \in\{1,2, \ldots, r\}$, thus it follows that

$$
\begin{gathered}
G_{N_{i_{k+1}, j_{k+1, r}}}(t)=\prod_{j=1}^{r} G_{N^{j} i_{k+1}, j_{k+1}}(t) \\
=\left(G_{N^{j_{i}}}(t)\right)^{r}=\left(\frac{\pi_{k+1} t^{\delta_{k+1}}}{1-\sum_{m=1}^{k} \pi_{m} t^{\delta_{m}}}\right)^{r}
\end{gathered}
$$

Theorem 1.8 Let $N_{i_{k+1}, j_{k+1}, r}$ be as in definition 1.6, then the probability $\left\{N_{i_{k+1}, j_{k+1}, r}=x\right\}$ is given by

$$
\begin{gathered}
f_{i_{k+1}, j_{k+1}, r}(x)=P\left(\begin{array}{c}
\left.N_{i_{k+1}, j_{k+1}, r}=x\right)= \\
x_{1}+x_{2}+\cdots+x_{k}+r-1 \\
x_{1}, x_{2}, \ldots, x_{k},+r-1
\end{array}\right) \times x_{k} ; i_{1}, i_{2}, \ldots, i_{k} j_{1}, j_{2}, \ldots, j_{k} \ni \\
p_{m=1}^{k} i_{m} x_{m}+\sum_{m=1}^{k} j_{m} x_{m}=x-\left(i_{k+1}-j_{k+1}\right) r \\
p^{x}\left(\frac{q}{p}\right)^{\sum_{m=1}^{k} j_{m} x_{m}+r j_{k+1}} ; x \geq\left(i_{k+1}+j_{k+1}\right) r \quad(1.10)
\end{gathered}
$$

Where the summation is taken over all non-negative integers $x_{1}, x_{2}, \ldots, x_{k} ; i_{1}, i_{2}, \ldots, i_{k} ; j_{1}, j_{2}, \ldots, j_{k}$ such that 


\section{Proof.}

$$
\sum_{m=1}^{k} i_{m} x_{m}+\sum_{m=1}^{k} j_{m} x_{m}=x-\left(i_{k+1}-j_{k+1}\right) r
$$

By theorem 1.7 the p.g.f. of $N_{i_{k+1}, j_{k+1, r}}$ is given by

Hence by binomial expansion we have that

$$
G_{N_{i_{k+1}, j_{k+1, r}}}(t)=\left(\frac{\pi_{k+1} t^{\delta_{k+1}}}{1-\sum_{m=1}^{k} \pi_{m} t^{\delta_{m}}}\right)^{r} ;|t| \leq 1, \pi_{m}=p^{i_{m}} q^{j_{m}}, \delta_{m}=i_{m}+j_{m}
$$

$$
\begin{aligned}
& G_{N_{i_{k+1}, j_{k+1, r}}}(t)=\left(\pi_{k+1} t^{\delta_{k+1}}\right)^{r} \sum_{x=0}^{\infty}\left(\begin{array}{c}
-r \\
x
\end{array}\right)\left(-\sum_{m=1}^{k} \pi_{m} t^{\delta_{m}}\right)^{x} \\
& =\left(\pi_{k+1} t^{\delta_{k+1}}\right)^{r} \sum_{\boldsymbol{x}=\mathbf{0}}^{\infty}\left(\begin{array}{c}
x+r-1 \\
r-1
\end{array}\right) \sum_{\substack{x_{1}, x_{2}, \ldots, x_{k} \ni \\
x_{1}+x_{2}+\cdots+x_{k}=x}}\left(\begin{array}{c}
x \\
x_{1}, x_{2}, \ldots, x_{k}
\end{array}\right) \prod_{m=1}^{k}\left(\pi_{m} t^{\delta_{m}}\right)^{x_{m}} \\
& =\left(p^{i_{k+1}} q^{j_{k+1}} t^{\delta_{k+1}}\right)^{r} \sum_{x=\mathbf{0}}^{\infty}\left(\begin{array}{c}
x+r-1 \\
r-1
\end{array}\right) \sum_{\substack{x_{1}, x_{2}, \ldots, x_{k} \ni \\
x_{1}+x_{2}+\cdots+x_{k}=x}}\left(\begin{array}{c}
x \\
x_{1}, x_{2}, \ldots, x_{k}
\end{array}\right) \prod_{m=1}^{k}\left(p^{i_{m}} q^{j_{m}} t^{\delta_{m}}\right)^{x_{m}} \\
& \left(p^{i_{k+1}} q^{j_{k+1}} t^{\delta_{k+1}}\right)^{r} \sum_{x=0}^{\infty} \sum_{x_{1}, x_{2}, \ldots, x_{k} ; i_{1}, i_{2}, \ldots, i_{k} ; j_{1}, j_{2}, \ldots, j_{k} \ni}\left(\begin{array}{c}
x+r-1 \\
r-1
\end{array}\right)\left(\begin{array}{c}
x \\
x_{1}, x_{2}, \ldots, x_{k}
\end{array}\right) \times \\
& \sum_{m=1}^{k} i_{m} x_{m}+\sum_{m=1}^{k} j_{m} x_{m}=x
\end{aligned}
$$

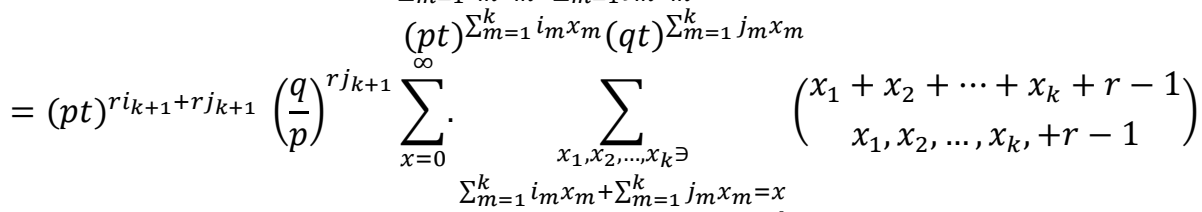

$$
\begin{aligned}
& \times(p t)^{\sum_{m=1}^{k} i_{m} x_{m}+\sum_{m=1}^{k} j_{m} x_{m}}\left(\frac{q}{p}\right)^{\sum_{m=1}^{k} j_{m} x_{m}} \\
& =\sum_{x=r i_{k+1}+r j_{k+1}}^{\infty} \sum_{\substack{x_{1}, x_{2}, \ldots, x_{k} ; i_{1}, i_{2}, \ldots, i_{k} ; j_{1}, j_{2}, \ldots, j_{k} \ni \\
\sum_{m=1}^{k} i_{m} x_{m}+\sum_{m=1}^{k} j_{m} x_{m}=x-r i_{k+1}+r j_{k+1}}}\left(\begin{array}{c}
x_{1}+x_{2}+\cdots+x_{k}+r-1 \\
x_{1}, x_{2}, \ldots, x_{k},+r-1
\end{array}\right) \times \\
& (p t)^{x}\left(\frac{q}{p}\right)^{\sum_{m=1}^{k} j_{m} x_{m}+r j_{k+1}} \\
& =\sum_{x=r i_{k+1}+r j_{k+1}}^{\infty} t^{x} P\left(N_{i_{k+1}, j_{k+1, r}}=x\right)
\end{aligned}
$$

Hence the result follows immediately.

Corollary1.9 Let $N_{i_{k+1}, j_{k+1}, r}$ be as in definition 1.6. If in particular we define

$$
\begin{gathered}
j_{m}=\left\{\begin{array}{l}
1, m=1,2, \ldots, k \\
0, m \geq k+1
\end{array} \text { and } \begin{array}{c}
i_{m}=m-1 . \\
P\left(N_{k, 0, r}=x\right)= \\
\sum_{\sum_{m=1}^{k}(m-1) x_{m}+\sum_{m=1}^{k} x_{m}=x-r k}^{x_{1}+x_{2}+\cdots+x_{k}+r-1} \\
p^{x}\left(\frac{q}{p}\right)^{\sum_{m=1}^{k} x_{m}}, \ldots, x_{k}, r-1
\end{array}\right) \times \\
; x \geq k r
\end{gathered}
$$

Then

Where the summation is taken over all non-negative integers $x_{1}, x_{2}, \ldots, x_{k} ;$ satisfying the condition $\sum_{m=1}^{k}(m-1) x_{m}+$ $\sum_{m=1}^{k} x_{m}=x-k r$.

Definition1.10. Let $N_{i_{k+1}, j_{k+1}, \mu}$ be a discrete random variables and $\mu$ a nonnegative integer constant. Assuming that $q \rightarrow 0$ and $r q \rightarrow \mu$ as $r \rightarrow \infty$. Then $N_{i_{k+1}, j_{k+1}, r} \rightarrow N_{i_{k+1}, j_{k+1}, \mu}$. We say that $N_{i_{k+1}, j_{k+1}, \mu}$ has the modified Poisson distribution of order $\left(i_{k+1}, j_{k+1}\right)$ denoted by $M P_{i_{k+1}, j_{k+1}}(. ; \mu)$.

Theorem 1.11 Let $N_{i_{k+1}, j_{k+1}, \mu}$ be as in definition 1.10, then the probability $\left\{N_{i_{k+1}, j_{k+1}, \mu}=x\right\}$ is given by

$$
\begin{gathered}
\lim _{r \rightarrow \infty} P\left(N_{i_{k+1}, j_{k+1}, r}-r i_{k+1}+r j_{k+1}=x\right) \rightarrow P\left(N_{i_{k+1}, j_{k+1}, \mu}=x\right)= \\
\sum_{\substack{x_{1}, x_{2}, \ldots, x_{k} ; i_{1}, i_{2}, \ldots, i_{k} ; j_{1}, j_{2}, \ldots, j_{k} \ni \\
\sum_{m=1}^{k} i_{m} x_{m}+\sum_{m=1}^{k} j_{m} x_{m}=x}} e^{-\mu i_{k+1}} \frac{\mu^{\sum_{m=1}^{k} j_{m} x_{m}}}{x_{1} !, x_{2} !, \ldots, x_{k} !} \forall i_{m} \geq 0
\end{gathered}
$$




\section{Proof.}

It suffices to show that the characteristic function of $N_{i_{k+1}, j_{k+1}, r}-r i_{k+1}+r j_{k+1}$ converges to $M P_{i_{k+1}, j_{k+1}}(. ; \mu)$ as $r \rightarrow \infty$. From (1.9) we have

$$
\phi_{N_{i_{k+1}, j_{k+1, r}}}(t)=\left(\frac{\pi_{k+1} \exp \left(\delta_{k+1} i t\right)}{1-\sum_{m=1}^{k} \pi_{m} \exp \left(\delta_{m} i t\right)}\right)^{r}
$$

Since $\left|q^{j_{k+1}} \exp \left(\delta_{k+1} i t\right)\right| \leq 1$ for $|t| \leq 1$. It then follows that

$$
\begin{gathered}
\phi_{N_{i_{k+1}}, j_{k+1, r}}(t) \leq\left(\frac{p^{i_{k+1}}}{1-\sum_{m=1}^{k} \pi_{m} \exp \left(\delta_{m} i t\right)}\right)^{r} \\
=\frac{\left(1-\frac{\mu}{r}\right)^{r i_{k+1}}}{\left(1-r^{-j_{m}} \sum_{m=1}^{k} p^{i_{m}} \mu^{j_{m}} \exp \left(\delta_{m} i t\right)\right)^{r}}
\end{gathered}
$$

By hypothesis, we have that $q \rightarrow 0$ and $r q \rightarrow \mu$ as $r \rightarrow \infty$. Thus, as $r \rightarrow \infty$ we have that

Hence the result follows immediately.

$$
\begin{aligned}
& \lim _{r \rightarrow \infty} \phi_{N_{i_{k+1}, j_{k+1}, r}}(t) \leq \lim _{r \rightarrow \infty} \frac{\left(1-\frac{\mu}{r}\right)^{r i_{k+1}}}{\left(1-r^{-j_{m}} \sum_{m=1}^{k} p^{i_{m}} \mu^{j_{m}} \exp \left(\delta_{m} i t\right)\right)^{r}} \\
&=\exp \left(-\mu i_{k+1}+\sum_{m=1}^{k} \mu^{j_{m}} \exp \left(\delta_{m} i t\right)\right)
\end{aligned}
$$

Corollary1.9 Let $N_{i_{k+1}, j_{k+1, \mu}}$ be as in definition 1.10. If in particular we define

Then

$$
j_{m}=\left\{\begin{array}{l}
1, m=1,2, \ldots, k \\
0, m \geq k+1
\end{array} \text { and } \quad i_{m}=m-1\right.
$$

$$
P\left(N_{i_{k+1}, 0, \mu}=x\right)=\sum_{\substack{x_{1}, x_{2}, \ldots, x_{k} \\ \sum_{m=1}^{k}(m-1) x_{m}+\sum_{m=1}^{k} x_{m}=x}} e^{-\mu k} \frac{\mu^{\sum_{m=1}^{k} x_{m}}}{x_{1} !, x_{2} !, \ldots, x_{k} !}
$$

\section{Conclusion}

We presented a new modified distribution function whose order of sequence of runs of success-failure is an arbitrary function of $k$, this modifies, generalize and complement the usual geometric distribution of order $k$ studied by several authors in the literature. The associated statistics, generating function and it relationship with some other discrete distributions using standard augment and convergence analysis are established.

\section{References}

Aki, S., (1985). Discrete distribution of order k on a binary sequence, Ann. Inst.Statist. Math. 36A, 205-224.

Aki, S. and Hirano, K. (1988). Some characteristics of the binomial distribution of order k and related distributions, Statistical Theory and Data Analysis II, Proceedings of the 2nd Pacific Area Statistical Conference, K. Matusita ed.,North-Holland, Amsterdam, 211-222.

Aki, S. and Hirano, K. (1989). Estimation of parameters in the discrete distributions of order k, Ann. Inst. Statist. Math., 41, 47-61.

Aki, S. and Hirano, K. (1996). Lifetime distribution and estimation problems of consecutive-k-out-of-n:F systems, Ann. Inst. Statist. Math., 48, 185-199.

Aki, S. and Hirano, K. (1997). Lifetime distributions of consecutive-k-out-of-n: F systems, Nonlinear Analysis, Theory, Methods Applications, 30, 555-562.

Aki, S. and Hirano, K. (1994). Distributions of numbers of failures and successes until the first consecutive k successes, Ann. Inst. Statist. Math., 46, 193-202.

Aki, S. and Hirano, K. (1995). Joint distributions of numbers of successruns and failures until the first consecutive k successes, Ann. Inst. Statist. Math., 47, 225-235.

Balakrishnan, N. (1997a). Joint distributions of numbers of success-runs and failures until the first consecutive k successes in a binary sequence, Ann. Inst. Statist. Math., 49, 519-529.

Chrysaphinou, O. and Papastavridis, S. G. (1990c). Reliability of a Consecutivek- out-of-n system in a random environment, J. Appl. Prob., 27, 452- 458.

Chiang, D. and Niu, S. C. (1981). Reliability of consecutive-k-out-of-n:F system, IEEE Transactions on Reliability, 30, April, 87-89.

Charalambides, Ch. A. (1994). Success runs in a circular sequence of independent

Bernoulli trials, Runs and Patterns in Probability: Selected Papers, Godbole, A. P. and Papastavridis, S. G. (Eds), 15-30, Kluwer.

Dhar, S. K. and Jiang, X. (1995) Probability bounds on the finite sum of the binary sequence of order k, J. Appl. Prob., 32, $1014-1027$.

Derman, C., Lieberman, G. J. and Ross, S. M. (1982). On the consecutivekout-of-n:F system, IEEE Transactions on Reliability, R-31, April, $57-63$.

Fu, J. C. (1985). Reliability of a large consecutive-k-out-of-n:F system, IEEE Trans.Reliability,vol.R-34,127-130.

Fu, J. C. (1986a). Bounds for reliability of large consecutive-k-out-of-n:F systems with unequal component reliability, IEEE Trans. Reliability, vol. R-35,316-319.

Fu, J. C. (1986b). Reliability of consecutive-k-out-of-n:F systems with (k-1)-step Markov Dependence, IEEE Trans. Reliability, vol. R-35, 602- 606. 
Fu, J. C. and Hu, Beihua (1987). On reliability of a large consecutive-koutof-n:F system with (k - 1)-step Markov dependence, IEEE Trans. Reliability,vol. R-36, 75-77.

Fu, J. C. and Koutras, M. V. (1994a). Poisson approximations for 2- dimensional patterns, Ann. Inst. Statist. Math., 46, $179-192$.

Fu, J. C. and Koutras, M. V. (1994b). Distribution theory of runs: A Markov chain approach, J. Amer. Statist. Assoc., 89, $1050-1058$.

Godbole, A. P. (1990a). Specific formulae for some success run distributions Statistics Probability Letters, 10, 119-124.

Godbole, A. P. (1991). Poisson approximations for runs and patterns of rare events, Adv. Appl. Prob., 23, 851-865.

Griffith, W. S. (1994). Runs and patterns with applications to reliability, Runs and Patterns in Probability: Selected Papers, Godbole, A. P. and Papastavridis, S. G. (Eds), 173-181, Kluwer.

Hirano, K. and Aki, S. (1993). On number of occurrences of success runs of specified length in a two-state Markov chain, Statistica Sinica, 3, 313-320.

Hirano, K. (1986). Some properties of the distributions of order k, Fibonacci Numbers and Their Applications, (eds. A.N. Philippou, G.E.Bergum and A.F.Horadam), 43-53,Reidel, Dordrecht.

Kontoleon, J. M. (1980). Reliability determination of r-successive-out-of-n: F system, IEEE Trans. Reliability, vol. R-29, 437.

Lambiris, M. and Papastavridis, S. (1985). Exact reliability formulas for linear circular consecutive-k-out-of-n:F systems, IEEE Trans. Reliability, vol.R-34, 124-126.

Makri, F. S. and Philippou, A. N. (1994). Binomial distributions of order K on the circle, Runs and Patterns in Probability: Selected Papers, Godbole, A.P. and Papastavridis, S.G. (Eds), 65-81, Kluwer.

Philippou, A. N., Georghiou, C. and Philippou, G. N. (1983). A generalized geometric distribution and some of its properties, Statistics Probability Letters, 1, 171-175.

Philippou, A. N. (1986). Distributions and Fibonacci polynomials of order k, longest runs, and reliability of consecutive-k-out-of-n:F system, Fibonacci Numbers and Their Applications, (eds. A. N. Philippou, G. E. Bergum and A.F. Horadam),203-227, Reidel,Dordrecht.

Papastavridis, S. (1987) A limit theorem for the reliability of a consecutivekout-of-n system, Adv. Appl. Prob., 19, 746-748.

Shao J. G. and Fu, S., (2015), Some generalisation of geometric distributin in Bernoulli trials by TPFG methods, WSEAS Transaction on Mathematics, 14(2). 146-155

Shao J. G. and Wang, Q. S., (2015), Multidimensional geometric distribution and Multidimensional poisson distribution in independent trials, Journal of Beijing Jiaotong University, 39 (3), 122-129.

\section{$\underline{\text { How to cite this article }}$}

Okoli, O.C. (2018). Modified Geometric Distribution of Certain Order and Some of its Properties. Tropical Journal of Applied Natural Sciences, 2(2): 143-149. Doi: https://doi.org/10.25240/TJANS.2018.2.2.19. 